\section{Self-organized growth of nanostructure arrays on strain-relief patterns}

\section{Harald Brune, Marcella Giovannini, Karsten Bromann \& Klaus Kern}

Institut de Physique Expérimentale, EPF Lausanne, CH-1015 Lausanne, Switzerland

The physical and chemical properties of low-dimensional structures depend on their size and shape, and can be very different from those of bulk matter. If such structures have at least one dimension small enough that quantum-mechanical effects prevail, their behaviour can be particularly interesting. In this way, for example, magnetic nanostructures can be made from materials that are non-magnetic in bulk ${ }^{1}$, catalytic activity can emerge from traditionally inert elements such as gold ${ }^{2}$, and electronic behaviour useful for device technology can be developed ${ }^{3,4}$. The controlled fabrication of ordered metal and semiconductor nanostructures at surfaces remains, however, a difficult challenge. Here we describe the fabrication of highly ordered, two-dimensional nanostructure arrays through nucleation of deposited metal atoms on substrates with periodic patterns defined by dislocations that form to relieve strain. The strain-relief patterns are created spontaneously when a monolayer or two of one material is deposited on a substrate with a different lattice constant. Dis-

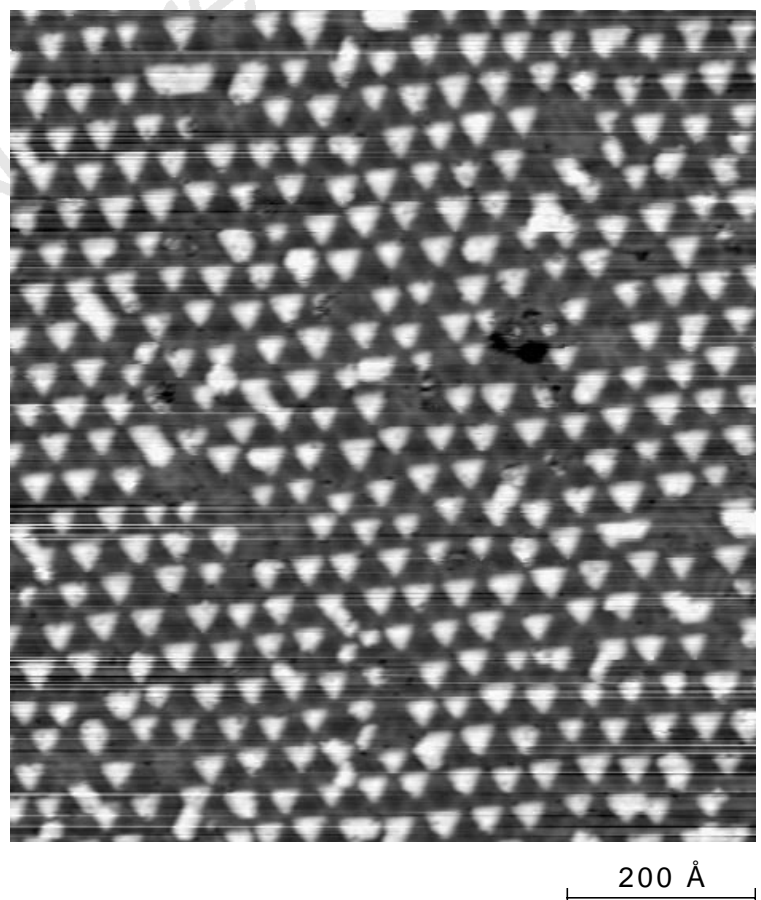

Figure 1 STM image of a periodic array of Fe islands nucleated on the dislocation network of a Cu bilayer on Pt(111) at $250 \mathrm{~K}$. locations often repel adsorbed atoms diffusing over the surface, and so they can serve as templates for the confined nucleation of nanostructures from adatoms. We use this technique to prepare ordered arrays of silver and iron nanostructures on metal substrates.

Arbitrary atomic-scalestructures can beassembled with thetip of a scanning tunnelling microscope (STM), either through direct displacement of adsorbed atoms ${ }^{5}$, or through tip-assisted decomposition of chemical species ${ }^{6}$. The principal drawback of methods based on scanning probes is their serial character. Approaches where a large number of structures can be created in parallel are selforganized growth - either in the kinetic ${ }^{7}$ or in the thermodynamic regime ${ }^{8-10}$ - and the controlled deposition of size-selected clusters from the gas phase ${ }^{11}$. Whereas the latter technique produces nanostructures of nearly uniform sizes on surfaces, the self-organized growth suffersfrom broad sizedistributions. In addition, both methods yield largely uncorrelated spatial distributions caused by the statistics of deposition and diffusion.

There have been several attempts to improve the spatial order in nanostructure growth. The vertical correlation of island nucleation in sequences of quantum dot and spacer layers yielded improved lateral order ${ }^{12}$. Also, misfit dislocations have been used for nanoscale structuring. Preferred nucleation of $\mathrm{Ni}$ at dislocations of the $\mathrm{Au}(111)$ reconstruction resulted in ordered islands ${ }^{13}$; for this system, the mechanism was identified as site-specific exchange, a finding that strongly reduces the number of elements suitable for thistype of ordering ${ }^{14}$. Also in semiconductors, island accumulation at dislocations has been reported ${ }^{15}$. However, there the bulk-like dislocations were not mobile enough to order into periodic patterns. Due to the attraction towards dislocations, islands were lined up in one dimension but were not periodic in two dimensions.

In heteroepitaxial systems that show strain relief through dislocations, the dislocations quite often arrange into highly ordered periodic patterns. This is due to mutual long-range repulsion and

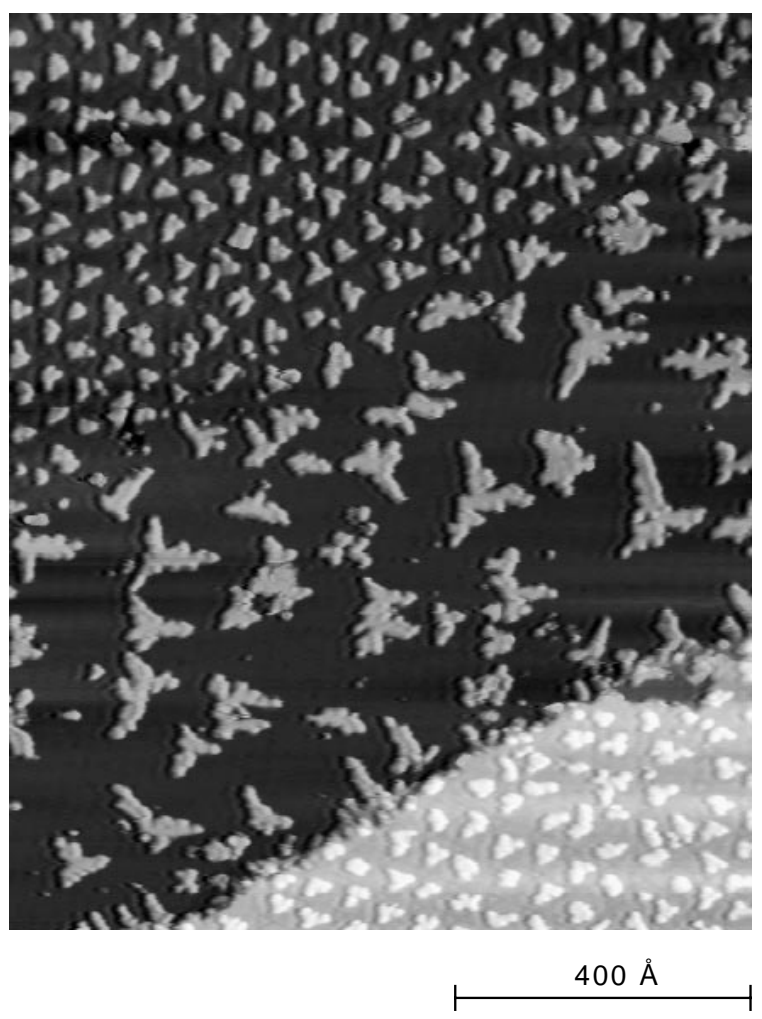

Figure 2 STM image of periodically ordered versus randomly nucleated islands on a heterogeneous substrate. The islands were grown by deposition of $0.1 \mathrm{ML}$ $\mathrm{Ag}$ at $110 \mathrm{~K}$ onto a Pt(111) surface pre-covered by $1.5 \mathrm{ML} \mathrm{Ag} \mathrm{(1} \mathrm{ML} \mathrm{is} \mathrm{one} \mathrm{adsorbed}$ atom per substrate atom). 
high mobility of the dislocations located at the surface. These surface dislocations are often strongly repulsive towards diffusing adatoms. Here we show that this can be used to transfer the dislocation periodicity through heterogeneous nucleation to regular superlattices of almost mono-dispersed islands. We demonstrate the feasibility of this self-organized growth method with $\mathrm{Fe}$ islands on the second monolayer (M L) of Cu on a Pt(111) substrate (Fig. 1), and with Ag nucleation on $2 \mathrm{ML}$ of Ag on Pt(111) (Figs 2 and 3). The array of Fe quantum dots in Fig. 1 shows the unique potential of the approach. The areal density of the Fe dots exceeds the densities at present achieved by electron-beam lithography by two orders of magnitude.

Random versus ordered nucleation are contrasted in the STM image shown in Fig. 2. Quite regularly spaced islands coexist with a part of the surface covered by randomly distributed islands. While the latter show the broad distribution of island size (Fig. 3c) and distance typically observed for nucleation on isotropic substrates, the former appear to be placed on a periodic grid. This marked difference is caused by the structure of the underlying substrate. Before growing the $\mathrm{Ag}$ islands, the $\mathrm{Pt}(111)$ substrate was pre- a

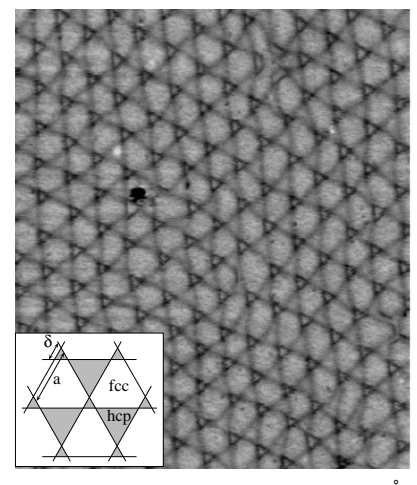

$200 \AA$

c

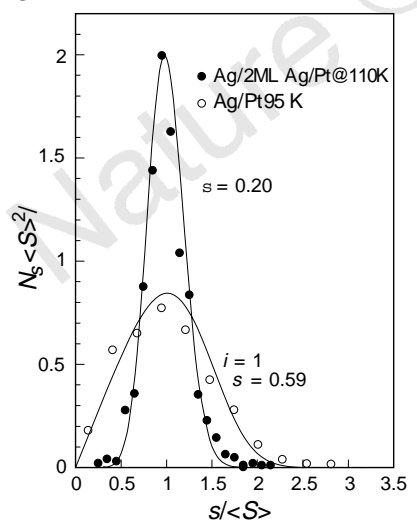

b
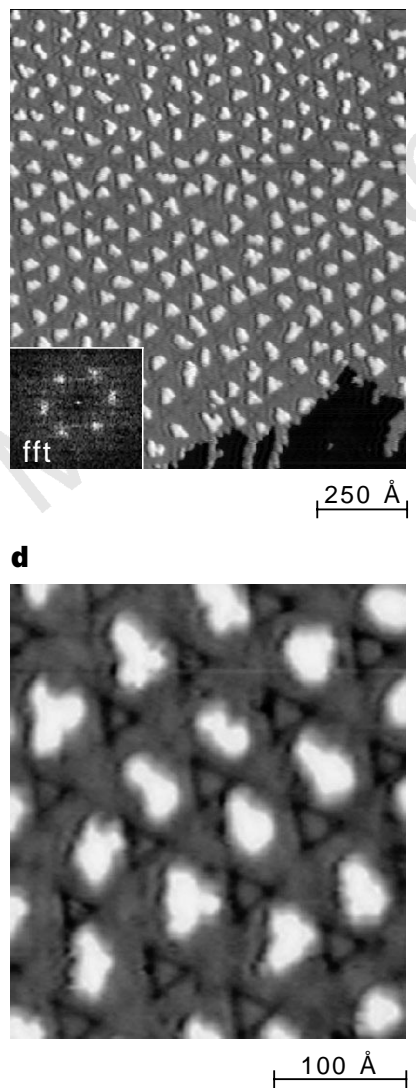

Figure 3 STM images showing the confined nucleation of adatom islands on a dislocation network. a, STM image of the ordered $(25 \times 25)$ dislocation network formed by the second $\mathrm{Ag}$ monolayer on $\mathrm{Pt}(111)$ on deposition at $400 \mathrm{~K}$ and subsequent annealing to $800 \mathrm{~K}$. The inset shows a model of this trigonal strainrelief pattern. $\mathbf{b}, \mathrm{A}$ superlattice of islands is formed on $\mathrm{Ag}$ deposition onto this network at $110 \mathrm{~K}$ (coverage $\Theta=0.10 \mathrm{ML}$ ). Inset, the Fourier transform of the STM image shows the high degree of order and the hexagonal symmetry of the nanostructure array. c, Island size distributions for random and ordered nucleation (curves, theory; dots, experiment). The curve for ordered nucleation is a binomial fit. The curve labelled $i=1$ shows the size distribution from scaling theory for random nucleation on an isotropic substrate. Size distributions were normalized according to scaling theory ( $s$ is the island size in atoms, $\langle S\rangle$ its mean value, and $N_{s}$ the density of islands with size $s$ per substrate atom). d, Detail of STM image $\mathbf{b}$. covered by $1.5 \mathrm{ML} \mathrm{Ag}$ such that the first and second monolayer coexist. These two layers differ in structure. The lattice constant of $\mathrm{Ag}$ is $4.3 \%$ larger than that of Pt. In the first monolayer, the $\mathrm{Ag}$ atoms are coherently strained by this amount and form a pseudomorphic layer ${ }^{16}$. As this layer is homogeneous (and isotropic), one observes random nucleation on top of it.

The second monolayer of Ag, on the other hand, forms a trigonal network of dislocations in which the compressive strain is partially relieved ${ }^{17}$. This network reveals a well established long-range order (Fig. 3a). Thedislocations mark transitions from face-centred cubic (f.c.c.) to hexagonal close-packed (h.c.p.) stacking. Larger areas with the energetically favoured f.c.c. stacking are achieved by displacing one class of domain walls (Fig. $3 a$ inset) relative to the crossing point of the two others. The resulting unit cell has trigonal symmetry and consists of a large quasi-hexagon (f.c.c.) and two triangles with opposite orientation (h.c.p. stacking).

Nucleation of Ag onto this network at low temperature leads to a high density of islands (Fig. 4), most of which are located away from dislocations. This implies that dislocations are repulsive barriers towards diffusing adatoms. At $T=110 \mathrm{~K}$, the island density approaches a stationary value of exactly one island per network unit cell (Fig. 3b). We note that all islands nucleate within the distorted hexagons (Fig. 3d). This implies preferential binding to f.c.c.-areas in agreement with theory ${ }^{18}$. O wing to this difference in binding energy, atoms landing on h.c.p. triangles can diffuse into the f.c.c. hexagons but not vice versa. The repulsive nature of the dislocations and the attraction towards specific sites within the unit cell are the key properties transferring the periodicity of the dislocation network to a highly ordered two-dimensional island superlattice.

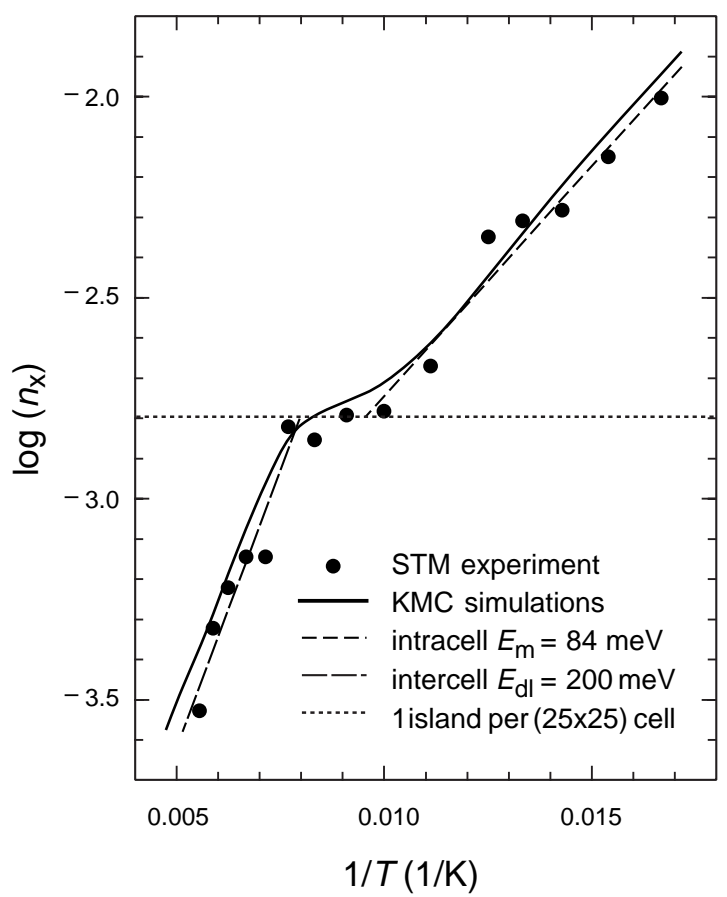

Figure 4 Arrhenius plot of measured (dots) and simulated (curve) island densities, $n_{x}$, for nucleation of $0.1 \mathrm{ML} \mathrm{Ag}$ on the dislocation network of Fig. 3a. The experimental island densities clearly show two slopes separated by a plateau with a density of one island per superstructure unit cell. The plateau is less pronounced in the Monte Carlo simulations than in the experiment. In the experiment, dislocations have a long-range repulsion towards adatoms, funnelling them to the unit-cell centre where the islands are nucleating. We did not attempt to simulate the complicated energy surface required to account for these findings. The islands in the simulation are therefore randomly placed within the unit cells; those located at the border sometimes overgrow a dislocation which smears out the plateau. 
The ordered nucleation is accompanied by an enhanced size uniformity (Fig. 3C). Atoms deposited onto a network unit cell stay confined to this cell by the surrounding dislocations. As all atoms arriving within one unit cell nucleate exactly one island there, the size distribution of these islands is binomial, $P_{k}=\left(\begin{array}{l}n \\ k\end{array}\right) p^{k} q^{n-k}$ (with $p=\Theta, q=1-\Theta, k$ being the island size, $n$ the size of the unit cell, and $\Theta$ the coverage). For our case of $p=0.1$ and $n=625$, the binomial distribution has a standard deviation of $\sigma=(q / n p)^{1 / 2}=$ 0.12 (normalized as in Fig. 3c). O wing to the convolution of tip and island shape there is a residual width explaining our larger experimental value of $\sigma=0.20$. This value represents an upper bound to the real size distribution. We note that $\sigma$ is often given for island diameters, where it is half the value of the island area distribution. Therefore our size distributions comparefavourably to state-of-theart results from self-organized quantum dots ${ }^{19}$.

Quantitative understanding of nucleation on the dislocation network is achieved through analysis of the Arrhenius behaviour of the saturation island density (Fig. 4). Thesystem can be described very well by a transition between two rate-limiting processes, the intra-cell diffusion between adjacent lattice sites at low temperatures, and the inter-cell diffusion across dislocations at high temperatures. To analyse the slopes in Fig. 4 in terms of activation energies for these diffusion processes, we performed kinetic Monte Carlo simulations on a hexagonal lattice with a $(25 \times 25)$ superstructure. For simplicity we did not distinguish between f.c.c. and h.c.p. sites, so the unit cell was a simple rhombus. The free parameters are the energy barriers for diffusion within $\left(E_{\mathrm{m}}\right)$ and across $\left(E_{\mathrm{dll}}\right)$ the unit cells. The simulation clearly shows the transition between two slopes. It yields $E_{\mathrm{m}}=84 \pm 8 \mathrm{meV}$ for intra-cell diffusion and $E_{\mathrm{dl}}=200 \pm 30 \mathrm{meV}$ for diffusion across dislocations. The strong repulsion at dislocations explains the extended temperature regime of ordered nucleation. These results are, to our knowledge, the first quantitative measurements of diffusion on an inhomogeneous substrate.

For our approach of confined nucleation to work, the dislocations must arrange into periodic patterns, and they must be repulsive for adatom diffusion. The first condition is met by numerous heteroepitaxial systems. Besides the presented examples, well ordered trigonal dislocation networks are found in a number of epitaxial metal systems ${ }^{20}$ and in semiconductors, for example, for surfactant-mediated growth of Geon Si(111) (ref. 21). The requirement for dislocations to be repulsive is found to be met by many metal systems where there is no exchange ${ }^{20}$. Also, moiré structures $^{22-24}$ could be appropriate for the present approach of selforganization. Like dislocation patterns, they reveal periodic variations in adatom binding energy.

Our experiments describe a method to create almost monodispersed, equally spaced nanostructures through self-organization on substrates with a periodic strain-relief pattern. Theinhomogeneous energy surface experienced by diffusing adatoms gives rise to heterogeneous nucleation and growth at specific sites within the superstructure, yielding well-ordered island superlattices. Our method may be useful for the growth of quantum arrays, that is, superlattices of nanostructures with size and period smaller than the Fermi wavelength.

Received 23 December 1997; accepted 23 April 1998.

1. Wildberger, K., Stepanyuk, V. S., Lang, P., Zeller, R. \& Dederichs, P. H. M agnetic nanostructures: $4 d$ clusters on Ag(001). Phys. Rev. Lett. 75, 509-512 (1995).

2. Haruta, M. Size and support-dependency in the catalysis of gold. Catal. Today 36, 153- 166 (1997)

3. Orlov, A. O., Amlani, I., Bernstein, G. H., Lent, C. S. \& Snider, G. L. Realization of a functional cell for quantum-dot cellular automata. Science 277, 928- 930 (1997).

4. Eberl, K. Quantum-dot lasers. Phys. World 10(9), 45-50 (1997).

5. Eigler, D. M . \& Schweizer, E. K. Positioning single atoms with a scanning tunnelling microscope Nature 344, 524- 526 (1990).

6. Kent, A. D., Molnar, S.v., Gider, S. \& Awschalom, D. D. Properties and measurement of scanning tunneling microscope fabricated ferromagnetic particle arrays. J. Appl. Phys. 76, 6656-6660 (1994).

7. Röder, H., Hahn, E., Brune, H., Bucher, J. P. \& Kern, K. Building one and two-dimensional nanostructures by diffusion-controlled aggregation at surfaces. Nature 366, 141-143 (1993).

8. Leonard, D., Krishnamurthy, M., Reaves, C. M., Denbaars, S. P. \& Petroff, P. M. Direct formation of quantum-sized dots from uniform coherent islands of InGaAs on GaAs-surfaces. Appl. Phys. Lett. 63, 3203-3205 (1993)

9. Nötzel, R., Tommyo, J. \& Tamamura, T. Self-organized growth of strained InGaAs quantum disks. Nature 369, 131-133 (1994).

10. M oison, J. M . et al. Self-organized growth of regular nanometer-scale InAs dots on GaAs. Appl. Phys. Lett. 64, 196- 198 (1994).

11. Bromann, K. et al. Controlled deposition of size selected Ag nanoclusters. Science 274, $956-958$ (1996)

12. Tersoff, J., Teichert, C. \& Lagally, M. G. Self-organization in growth of quantum dot superlattices. Phys. Rev. Lett. 76, 1675- 1678 (1996).

13. Chambliss, D. D., Wilson, R. J. \& Chiang, S. Nucleation of ordered Ni island arrays on $\mathrm{Au}(111)$ by surface-lattice dislocations. Phys. Rev. Lett. 66, 1721-1724 (1991).

14. M eyer, J. A., Baikie, J. D., Kopatzki, E. \& Behm, R. J. Preferential island nucleation at the elbows of the $\mathrm{Au}(111)$ herringbone reconstruction through place exchange. Surf. Sci. 365, L647- L651 (1996).

15. Shiryaev, S. Y., Jensen, F., H ansen, J. L., Petersen, J. W. \& Larsen, A. N. Nanoscale structuring by misfit dislocations in $\mathrm{Si}_{1-x} \mathrm{Ge}_{\mathrm{x}} / \mathrm{Si}$ epitaxial systems. Phys. Rev. Lett. 78, 503- 506 (1997).

16. Brune, H. et al. The effect of strain on surface diffusion and nucleation. Phys. Rev. B 52, R14380R14383 (1995).

17. Brune, H., Röder, H., Boragno, C. \& Kern, K. Strain relief at hexagonal close packed interfaces. Phys. Rev. B 49, 2997- 3000 (1994).

18. Ratsch, C., Seitsonen, A. P. \& Scheffler, M. Strain-dependence of surfacediffusion: Ag on Ag(111) and Pt(111). Phys. Rev. B 55, 6750-6753 (1997).

19. Fafard, S., Leon, R., Leonard, D., M arz, J. L. \& Petroff, P. M. Phonons and radiative recombination in self-assembled quantum dots. Phys. Rev. B 52, 5752-5755 (1995)

20. Brune, H. Microscopic view of epitaxial metal growth: ... . Surf. Sci. Rep. 31, 121-229 (1998).

21. Horn-von-H oegen, M., Falou, A. A., Pietsch, H., Müller, B. H. \& Henzler, M. Formation of the interfacial dislocation network in surfactant-mediated growth of Ge on Si(111) by SPALEED Part I. Surf. Sci. 298, 29- 42 (1993)

22. Wiederholt, T., Brune, H., Wintterlin, J., Behm, R. J. \& Ertl, G. Formation of two-dimensional sulfide phases on Al(111): an STM study. Surf. Sci. 324, 91- 105 (1994).

23. Besenbacher, F., Nielsen, L. P. \& Sprunger, P. T. in Growth and Properties of Ultrathin Epitaxial Layers (eds King, D. A. \& Woodruff, D. P.) 207-257 (Elsevier, Amsterdam, 1997).

24. Böhringer, M., Jiang, Q., Berndt, R., Schneider, W. D. \& Zegenhagen, J. Discommensurations, epitaxial growth and island formation in Cu/Ge(111). Surf. Sci. 367, 245- 260 (1996).

Acknowledgements. We thank J. Jacobsen, K. Jacobsen and J. Nørskov for providing us with the kinetic Monte Carlo computer code that we adopted for nucleation on a superlattice.

Correspondence and requests for materials should be addressed to K.K. 